\title{
A Strategy to Employ Clitoria ternatea as a Prospective Brain Drug Confronting Monoamine Oxidase (MAO) Against Neurodegenerative Diseases and Depression
}

\author{
A. Anita Margret • T. Nargis Begum • \\ S. Parthasarathy $\cdot$ S. Suvaithenamudhan
}

Received: 14 October 2015/Accepted: 18 November 2015/Published online: 14 December 2015

(C) The Author(s) 2015. This article is published with open access at Springerlink.com

\begin{abstract}
Ayurveda is a renowned traditional medicine practiced in India from ancient times and Clitoria ternatea is one such prospective medicinal herb incorporated as an essential constituent in a brain tonic called as medhya rasayan for treating neurological disorders. This work emphasises the significance of the plant as a brain drug there by upholding Indian medicine. The phytochemicals from the root extract were extricated using gas chromatography-mass spectrometry assay and molecular docking against the protein Monoamine oxidase was performed with four potential compounds along with four reference compounds of the plant. This persuades the prospect of $C$. ternatea as a remedy for neurodegenerative diseases and depression. The in silico assay enumerates that a major compound (Z)-9,17-octadecadienal obtained from the chromatogram with a elevated retention time of 32.99 furnished a minimum binding affinity energy value of $-6.5 \mathrm{kcal} / \mathrm{mol}$ against monoamine oxidase (MAO-A). The interactions with the amino acid residues ALA 68, TYR 60 and TYR 69 were analogous to the reference compound kaempferol-3-monoglucoside with a least score of $-13.90 /-12.95 \mathrm{kcal} / \mathrm{mol}$ against the isoforms (MAO) A and B. This study fortifies the phytocompounds of C. ternatea as MAO-inhibitors and to acquire a pharmaceutical approach in rejuvenating Ayurvedic medicine.
\end{abstract}

\footnotetext{
A. A. Margret $(\square)$

Department of Biotechnology and Bioinformatics, Bishop Heber

College, Tiruchirappalli 620017, India

e-mail: anitamargret@gmail.com

T. N. Begum

Department of Biotechnology, Jamal Mohamed College,

Tiruchirappalli 620020, India

S. Parthasarathy $\cdot$ S. Suvaithenamudhan

Department of Bioinformatics, School of Life Sciences,

Bharathidasan University, Tiruchirappalli 620024, India
} 
Graphical Abstract

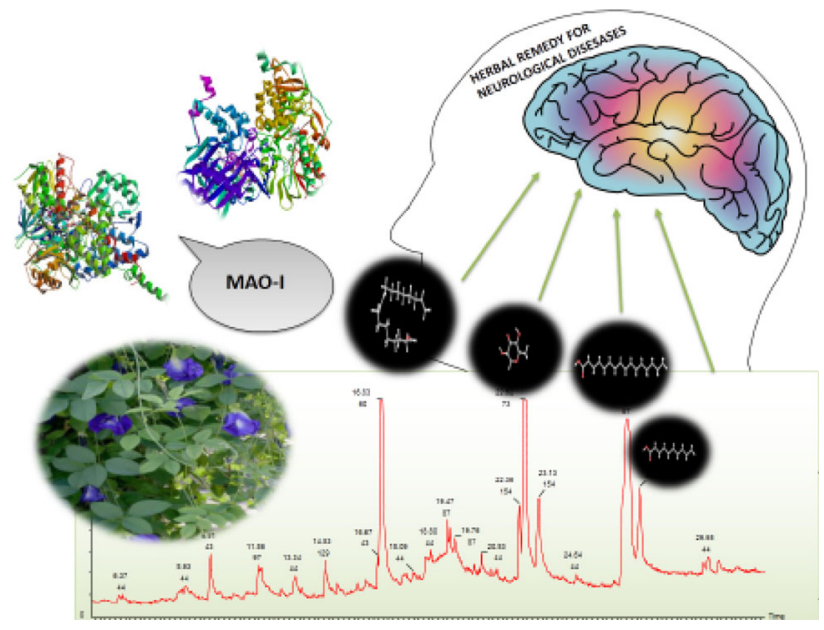

Keywords (Z)-9,17-Octadecadienal $\cdot$ Kaempferol-3-monoglucoside $\cdot$ Monoamine oxidase $\cdot$ Clitoria ternatea $\cdot$ Molecular docking $\cdot$ Ayurvedic medicine

\section{Introduction}

Alternative medicines disburse a new stratagem in restoring health and traditional medicines like Ayurveda has an embedded background and its patrons are drawn from ancient communities. The antiquity seized by ayurvedic medicine has formulated treatises to enhance mental health that promote memory and intelligence [1]. Medhya drugs constitute an assemblage of medicinal herbs that acts on the nervous system and improve mental abilities [2]. Medhya rasayana is a brain and nervine tonic that has been used for centuries in rejuvenating neurons and treating neurological diseases [3, 4]. Shankhpushpi is a reputed drug constituted in medhya rasayana whose name is derived from Sanskrit language. It illustrates a plant with flowers shaped like a conch or shankha which is a sacred instrument used in ritual worships. According to the pharmacopoeia of India, Convolvulus pluricaulis (Convolvulaceae) as a whole plant rightfully claim the name of Shankapushpi but, Ayurvedic practitioners have used three other medicinal herbs such as Evolvulus alsinoides Linn. (Convolvulaceae), Canscora decussata Schult. (Gentianaceae) and Clitoria ternatea Linn. (Papilionaceae) [5-9].

Though all the four plants are catalogued under the same class Magnoliopsida, C. ternatea separates itself at the level of sub-class belonging to Rosidae, while the other three belong to Asteridae. Clitoria ternatea, is distinct among other herbs and has the property of being a good brain drug [10]. Therefore there is a sturdy instinct to evaluate the phytoconstituents of the plant to treat mental disorders. A wide range of phytocompounds including ternatins, alkaloids, flavonoids, saponins, tannins, carbohydrates, proteins, resins, starch, taraxerol, taraxerone and secondary metabolites such as triterpenoids, flavonol glycosides, anthocyanins, steroid elevates the hope of endorsing $C$. ternatea as an efficient botanical medicine combating neurological ailments. This study exonerates the phytocompounds present in the root extract of the plant with a gas chromatography-mass spectrometry assay pursued by a molecular docking against a flavoenzyme Monoamine oxidase (MAO).

This protein is responsible for the oxidative deamination of neurotransmitter and dietary amines [11-13]. This enzyme degrades neurotransmitters such as serotonin and dopamine in the brain which is coded for by the MAOA gene [14-16]. Neurotransmitters play a pivotal role in mood, arousal, and emotions, even affecting impulse control.

The isoforms of Monoamine Oxidase (A and B) are categorised based on their substrate preference and inhibitor selectivity. Inhibitors of MAO-A are clinically used as antidepressants and anxiolytics [17, 18] while MAO-B 
inhibitors are used for the treatment of Parkinson's disease and for symptoms associated with Alzheimer's disease [19, 20].

Although several synthetic monoamine oxidase inhibitor (MAOI) have emerged as antidepressant drugs, the desire of herbal medicine is excessive. They are proficient in surpassing the adverse effects and improves a better sustainability. Hence a substantial study establishing $C$. ternatea as monoamine oxidase inhibitor (MOAI) fetches a stoppage solution against depression and neurological problems which hoists Ayurveda extensively.

\section{Results and Discussion}

Phytochemical assay of the plant $C$. ternatea was performed to divulge the essential phytocompounds which draw a base line in accessing their medicinal significance. The roots of the plant have an extended antiquity to promote mental power memory retention and alleviate psychotic stress [21]. Studies have revealed that aqueous root extract of $C$. ternatea enhances memory in rats while, alcoholic extracts of aerial and root parts of $C$. ternatea attenuated electroshock-induced amnesia [22, 23]. Hence this study determined root as the vital part that reveals the utmost essential phytocompounds.

\subsection{Extraction of Volatile Phytochemicals Augmenting Brain Function by GC-MS Assay}

GC-MS chromatogram analysis pertained from the ethanol extract of $C$. ternatea (Fig. 1) extricated twenty-five different compounds illustrated with twenty intense peaks indicating the presence of these phytochemical in a high constituent. The phytocompounds were detected and catalogued in parallel to the NIST library (Table 1). Among them, the most prevailing compounds are n-hexadecanoic acid $(21.32 \%)$ and (Z)-9,17-Octadecadienal $(28.76 \%)$, with a retention time of 22.62 and $26.73 \mathrm{~min}$. D-Allose (17.53\%), pyrrolo[1,2-a]pyrazine-1,4-dione (5.5\%), and 2,3-dihydro-3,5dihydroxy-6-methyl-4H-pyran-4-one $(3.76 \%)$ pursue the former compounds with a retention time of 16.83, 23.13 and $9.91 \mathrm{~min}$. The foremost compound (Z)-9,17-octadecadienal is categorized as aldehyde and commonly called as linolenic acid. It is an essential omega-3 fatty acid that has an explicit therapeutic value in regulating cholesterol level in blood. Reports unveil that they have neuroprotective properties and increased intake of $\alpha$-linolenic acid reduced depressive symptoms thereby maintaining robust mental health mental health [24-26]. Furthermore, palmitic acid (n-hexadecanoic acid) is one another indigenous compound which has an antioxidant property and acts as a 5-alpha reductase inhibitor [27]. Studies report that it has anticonvulsant and antidepressant property. The scavenging and inhibition of free radicals, inhibits the neurotoxicity of amyloid- $\beta$ thereby offering protection against hypoxic challenges [28-30]. A diverse range of flavonoids occurs in traditional medicine that exert as sedatives and carry out anxiolytic effects. This is due to the cognitive enhancement of the up regulation of cholinergic that results in the binding of GABAA receptors [29, 30]. This results in the inhibition of monoamine oxidase thereby raising the level of noradrenalin [31]. 2,3-Dihydro-3,5-dihydroxy-6-methyl-4H-pyran-4-one is characterized as a fragment of flavanoids which when synthesised by plants, are termed as phytoestrogens. Epidemiological studies suggests that they have the potentials of treating neurologic disorders such as dementia and Alzheimer's disease [32, 33]. Hence the analysis of GC-MS further extends a need of study to assay the antidepressant and neuroprotective aspect of the major compounds extricated from the root extract.

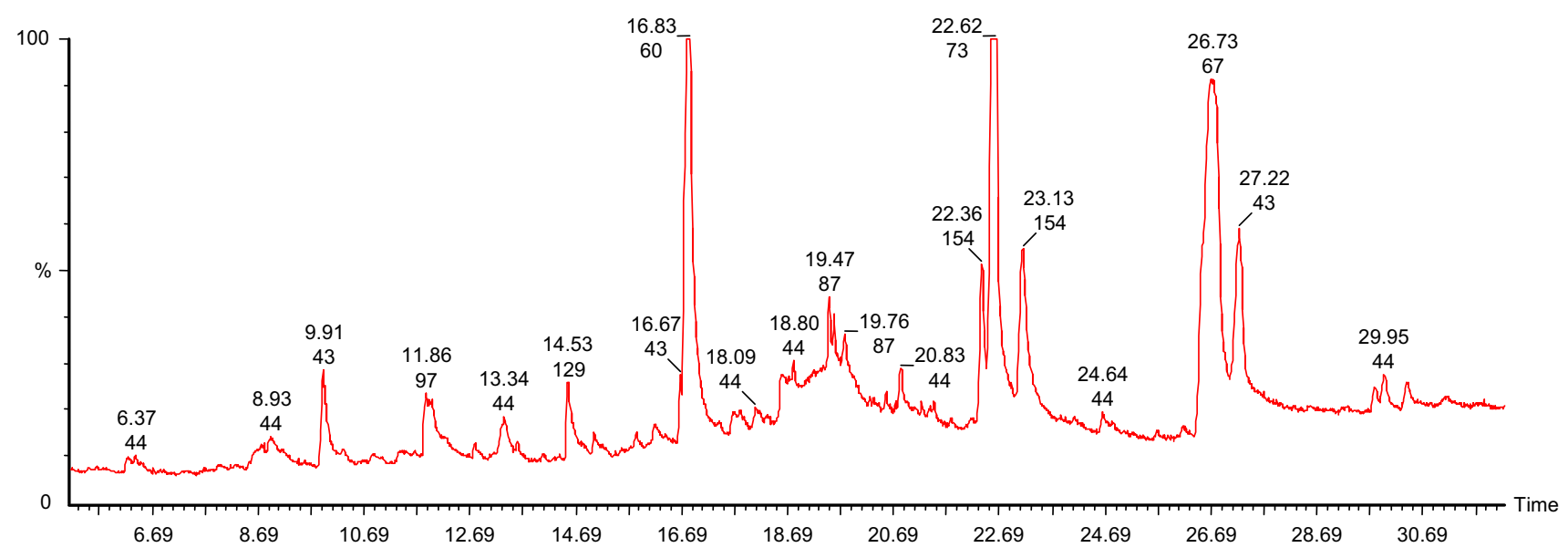

Fig. 1 A chromatogram illustrating the presence of bioactive phytochemicals 
Table 1 List of extricated compounds attained from GC-MS Assay

\begin{tabular}{|c|c|c|c|c|}
\hline S. no. & Peak name & Retention time & Peak area & $\%$ Peak area \\
\hline 1. & $\begin{array}{l}\text { Name: 2-Furancarboxaldehyde, 5-methyl- } \\
\text { Formula: } \mathrm{C}_{6} \mathrm{H}_{6} \mathrm{O}_{2} \\
\text { MW: } 110\end{array}$ & 6.23 & 1485494 & 0.4220 \\
\hline 2. & $\begin{array}{l}\text { Name: 2,4-Dihydroxy-2,5-dimethyl-3(2H)-furan-3-one } \\
\text { Formula: } \mathrm{C}_{6} \mathrm{H}_{8} \mathrm{O}_{4} \\
\text { MW: } 144\end{array}$ & 6.37 & 1298819 & 0.3689 \\
\hline 3. & $\begin{array}{l}\text { Name: Pyrrolidine, 3-methyl- } \\
\text { Formula: } \mathrm{C}_{5} \mathrm{H}_{11} \mathrm{~N} \\
\text { MW: } 85\end{array}$ & 8.93 & 2331724 & 0.6623 \\
\hline 4. & $\begin{array}{l}\text { Name: 4H-Pyran-4-one, 2,3-dihydro-3,5-dihydroxy-6-methyl- } \\
\text { Formula: } \mathrm{C}_{6} \mathrm{H}_{8} \mathrm{O}_{4} \\
\text { MW: } 144\end{array}$ & 9.91 & 13264328 & 3.767 \\
\hline 5. & $\begin{array}{l}\text { Name: Uracil } \\
\text { Formula: } \mathrm{C}_{4} \mathrm{H}_{4} \mathrm{~N}_{2} \mathrm{O}_{2} \\
\text { MW: } 112\end{array}$ & 11.39 & 2594699 & 0.7370 \\
\hline 6. & $\begin{array}{l}\text { Name: Cyclohexanone,2-isopropyl-2,5-dimethyl- } \\
\text { Formula: } \mathrm{C}_{11} \mathrm{H}_{20} \mathrm{O} \\
\text { MW: } 168\end{array}$ & 11.85 & 3496772 & 0.9933 \\
\hline 7. & $\begin{array}{l}\text { Name: 2-Methoxy-4-vinylphenol } \\
\text { Formula: } \mathrm{C}_{9} \mathrm{H}_{10} \mathrm{O}_{2} \\
\text { MW: } 150\end{array}$ & 12.78 & 1121110 & 0.3185 \\
\hline 8. & $\begin{array}{l}\text { Name: Valeric acid, 2,3-epoxy-3,4-dimethyl-, tert-butyl ester, cis- } \\
\text { Formula: } \mathrm{C}_{11} \mathrm{H}_{20} \mathrm{O}_{3} \\
\text { MW: } 200\end{array}$ & 13.34 & 8129658 & 2.309 \\
\hline 9. & $\begin{array}{l}\text { Name: n-Decanoic acid } \\
\text { Formula: } \mathrm{C}_{10} \mathrm{H}_{20} \mathrm{O}_{2} \\
\text { MW: } 172\end{array}$ & 13.58 & 1971583 & 0.5600 \\
\hline 10. & $\begin{array}{l}\text { Name: 2-Butenoic acid, 4,4-dimethoxy-, methyl ester } \\
\text { Formula: } \mathrm{C}_{7} \mathrm{H}_{12} \mathrm{O}_{4} \\
\text { MW: } 160\end{array}$ & 14.53 & 6979632 & 1.982 \\
\hline 11. & $\begin{array}{l}\text { Name: Phenol, 2-methoxy-4-(1-propenyl)-, (E)- } \\
\text { Formula: } \mathrm{C}_{10} \mathrm{H}_{12} \mathrm{O}_{2} \\
\text { MW: } 164\end{array}$ & 15.04 & 1273167 & 0.3616 \\
\hline 12. & $\begin{array}{l}\text { Name: 5-n-Propylhydantoin } \\
\text { Formula: } \mathrm{C}_{6} \mathrm{H}_{10} \mathrm{~N}_{2} \mathrm{O}_{2} \\
\text { MW: } 142\end{array}$ & 16.19 & 731662 & 0.2078 \\
\hline 13. & $\begin{array}{l}\text { Name: D-Allose } \\
\text { Formula: } \mathrm{C}_{6} \mathrm{H}_{12} \mathrm{O}_{6} \\
\text { MW: } 180\end{array}$ & 16.83 & 61745524 & 17.53 \\
\hline 14. & $\begin{array}{l}\text { Name: Benzoic acid, 4-hydroxy-3-methoxy- } \\
\text { Formula: } \mathrm{C}_{8} \mathrm{H}_{8} \mathrm{O}_{4} \\
\text { MW: } 168\end{array}$ & 17.66 & 1914401 & 0.5438 \\
\hline 15. & $\begin{array}{l}\text { Name: 1,6-Anhydro-á-D-glucofuranose } \\
\text { Formula: } \mathrm{C}_{6} \mathrm{H}_{10} \mathrm{O}_{5} \\
\text { MW: } 162\end{array}$ & 18.58 & 1833479 & 0.5208 \\
\hline 16. & $\begin{array}{l}\text { Name: Phenol, 2,6-dimethoxy-4-(2-propenyl)- } \\
\text { Formula: } \mathrm{C}_{11} \mathrm{H}_{14} \mathrm{O}_{3} \\
\text { MW: } 194\end{array}$ & 18.80 & 829054 & 0.2355 \\
\hline
\end{tabular}


Table 1 continued

\begin{tabular}{|c|c|c|c|c|}
\hline S. no. & Peak name & Retention time & Peak area & $\%$ Peak area \\
\hline 17. & $\begin{array}{l}\text { Name: Tetradecanoic acid } \\
\text { Formula: } \mathrm{C}_{14} \mathrm{H}_{28} \mathrm{O}_{2} \\
\text { MW: } 228\end{array}$ & 19.47 & 2008953 & 0.5706 \\
\hline 18. & $\begin{array}{l}\text { Name: Phenol, 4-(3-hydroxy-1-propenyl)-2-methoxy- } \\
\text { Formula: } \mathrm{C}_{10} \mathrm{H}_{12} \mathrm{O}_{3} \\
\text { MW: } 180\end{array}$ & 19.76 & 2250140 & 0.6392 \\
\hline 19. & $\begin{array}{l}\text { Name: 1,13-Tetradecadien-3-one } \\
\text { Formula: } \mathrm{C}_{14} \mathrm{H}_{24} \mathrm{O} \\
\text { MW: } 208\end{array}$ & 20.55 & 1048599 & 0.2979 \\
\hline 20. & $\begin{array}{l}\text { Name: 1,6-Hexanediamine, 2,2,4-trimethyl- } \\
\text { Formula: } \mathrm{C}_{9} \mathrm{H}_{22} \mathrm{~N}_{2} \\
\text { MW: } 158\end{array}$ & 20.82 & 1607754 & 0.4567 \\
\hline 21. & $\begin{array}{l}\text { Name: Pyrrolo[1,2-a]pyrazine-1,4-dione, hexahydro-3-(2-methylpropyl)- } \\
\text { Formula: } \mathrm{C}_{11} \mathrm{H}_{18} \mathrm{~N}_{2} \mathrm{O}_{2} \\
\text { MW: } 210\end{array}$ & 22.36 & 13352407 & 3.792 \\
\hline 22. & $\begin{array}{l}\text { Name: } n \text {-Hexadecanoic acid } \\
\text { Formula: } \mathrm{C}_{16} \mathrm{H}_{32} \mathrm{O}_{2} \\
\text { MW: } 256\end{array}$ & 22.62 & 75068920 & 21.32 \\
\hline 23. & $\begin{array}{l}\text { Name: Pyrrolo[1,2-a]pyrazine-1,4-dione, hexahydro-3-(2-methylpropyl)- } \\
\text { Formula: } \mathrm{C}_{11} \mathrm{H}_{18} \mathrm{~N}_{2} \mathrm{O}_{2} \\
\text { MW: } 210\end{array}$ & 23.13 & 19559404 & 5.555 \\
\hline 24. & $\begin{array}{l}\text { Name: } 9,17-\text { Octadecadienal, (Z)- } \\
\text { Formula: } \mathrm{C}_{18} \mathrm{H}_{32} \mathrm{O} \\
\text { MW: } 264\end{array}$ & 26.73 & 101281232 & 28.76 \\
\hline 25. & $\begin{array}{l}\text { Name: Octadecanoic acid } \\
\text { Formula: } \mathrm{C}_{18} \mathrm{H}_{36} \mathrm{O}_{2} \\
\text { MW: } 284\end{array}$ & 27.22 & 22540484 & 6.402 \\
\hline 26. & $\begin{array}{l}\text { Name: } 13 \text {-Octadecenal, (Z)- } \\
\text { Formula: } \mathrm{C}_{18} \mathrm{H}_{34} \mathrm{O} \\
\text { MW: } 266\end{array}$ & 29.77 & 2328958 & 0.6615 \\
\hline
\end{tabular}

\subsection{Molecular Docking Studies}

An in silico assay was done to determine the best compound by docking against the depression and neurodegenerative inducing protein MOA (Mono Amine Oxidase). Alterations in monoaminergic transmission are reported to be related with the instigation of neurodegenerative diseases such as Parkinson's, Alzheimer's diseases and psychiatric disorders such as depression and anxiety [34]. In the present study, four phytocompounds confiscated from the root extracts of $C$. ternatea were docked computationally into the active site of the monoamine oxidase isoforms (MAO-A and MAO-B) and were investigated to endorse their inhibitory potency. Former studies deem to have the presence of imperative primary and secondary metabolites from the whole plant that are medically significant. Combined chemical analysis have isolated many vital phytocompounds such as kaempferol (kaempferol 3-neohesperidoside, kaempferol 3-rutinoside, kaempferol 3-glucoside), quercetin (quercetin 3-2 G-rhamnosyl rutinoside, quercetin 3-neohesperidoside, Quercetin 3-rutinoside, quercetin 3-glucoside), Myricetin 3-neohesperidoside, Myricetin 3-rutinoside, Myricetin 3-glucoside and delphinidin 3,3,5-triglucoside [35-38]. Conversely, there is a need of a study to explicit these phytocompounds against a target as obstacle. Hence four reference 
Table 2 Hit list of the ligands with the target protein MAO-A

\begin{tabular}{|c|c|c|c|c|c|}
\hline S. no & Compound & $\begin{array}{l}\text { Binding score } \\
\text { energy value } \\
\text { (Kcal/mol) }\end{array}$ & $\begin{array}{l}\text { No. of hydrogen } \\
\text { bonds }\end{array}$ & $\begin{array}{l}\text { Hydrogen } \\
\text { bond length }\end{array}$ & $\begin{array}{l}\text { Interacting } \\
\text { amino acid } \\
\text { residue }\end{array}$ \\
\hline 1. & $\begin{array}{l}\text { Name: kaempferol-monoglucoside } \\
\text { Formula: } \mathrm{C}_{21} \mathrm{H}_{20} \mathrm{O}_{1} \\
\text { MW: } 448.3769 \\
\text { Pub Chem ID: } 5282102\end{array}$ & -13.9077 & 7 & $\begin{array}{l}2.05 \\
2.19 \\
2.06 \\
1.57 \\
2.41 \\
1.88 \\
2.02\end{array}$ & $\begin{array}{l}\text { ALA } 68 \\
\text { GLN443 } \\
\text { GLN66 } \\
\text { GLN443 } \\
\text { MET445 } \\
\text { TYR69 } \\
\text { ASN181 }\end{array}$ \\
\hline 2. & $\begin{array}{l}\text { Name: Quercetin } \\
\text { Formula: } \mathrm{C}_{20} \mathrm{H}_{40} \mathrm{O} \\
\text { MW: } 296.531 \\
\text { Pub Chem ID: } 5280343\end{array}$ & -11.4246 & 2 & $\begin{array}{l}2.17 \\
2.16\end{array}$ & $\begin{array}{l}\text { ASN } 181 \\
\text { PHE } 208\end{array}$ \\
\hline 3. & $\begin{array}{l}\text { Name: Malvidin-3-0-glucoside } \\
\text { Formula: } \mathrm{C}_{23} \mathrm{H}_{2}{ }_{5} \mathrm{O}_{12} \\
\text { MW: } 493.4374 \\
\text { Pub Chem ID: } 443652\end{array}$ & -7.64073 & 3 & $\begin{array}{l}2.32 \\
2.25 \\
2.15\end{array}$ & $\begin{array}{l}\text { ALA } 68 \\
\text { TYR } 69 \\
\text { GLN215 }\end{array}$ \\
\hline 4. & $\begin{array}{l}\text { Name: Delphinidin-3,5-diglucoside } \\
\text { Formula: } \mathrm{C}_{27} \mathrm{H}_{31} \mathrm{O}_{17} \\
\text { MW: } 627.52484 \\
\text { Pub Chem ID: } 10100906\end{array}$ & - & - & - & - \\
\hline 5. & $\begin{array}{l}\text { Name: } 9,17 \text { Octadecadienal,(Z)- } \\
\text { Formula: } \mathrm{C}_{18} \mathrm{H}_{32} \mathrm{O} \\
\text { MW: } 264 \\
\text { Pub Chem ID: } 5365667\end{array}$ & -6.59394 & 2 & $\begin{array}{l}1.86 \\
1.87\end{array}$ & $\begin{array}{l}\text { ALA } 68 \\
\text { TYR } 69\end{array}$ \\
\hline 6. & $\begin{array}{l}\text { Name: 4H-Pyran-4-one, 2,3-dihydro- } \\
\text { 3,5-dihydroxy-6-methyl- } \\
\text { Formula: } \mathrm{C}_{6} \mathrm{H}_{8} \mathrm{O}_{4} \\
\text { MW: } 144 \\
\text { Pub Chem ID: } 119838\end{array}$ & -6.21223 & 1 & 1.72 & PHE208 \\
\hline 7. & $\begin{array}{l}\text { Name: } n \text {-Hexadecanoic acid } \\
\text { Formula: } \mathrm{C}_{16} \mathrm{H}_{32} \mathrm{O}_{2} \\
\text { MW: } 256 \\
\text { Pub Chem ID: } 985\end{array}$ & -5.06337 & 3 & $\begin{array}{l}2.21 \\
2.05 \\
2.10\end{array}$ & $\begin{array}{l}\text { ALA } 68 \\
\text { ALA } 68 \\
\text { MET } 445\end{array}$ \\
\hline & $\begin{array}{l}\text { 8. Name: n-Decanoic acid } \\
\text { Formula: } \mathrm{C}_{10} \mathrm{H}_{20} \mathrm{O}_{2} \\
\text { MW: } 172 \\
\text { Pub Chem ID: } 2969\end{array}$ & -4.03859 & 3 & $\begin{array}{l}2.13 \\
2.16 \\
1.96\end{array}$ & $\begin{array}{l}\text { MET } 445 \\
\text { TYR } 69 \\
\text { ALA } 68\end{array}$ \\
\hline
\end{tabular}

compounds (kaempferol-3-monoglucoside, Delphinidin3,5-diglucoside, Malvidin-3-O-glucoside and Quercetin) were elected to endure docking studies, comparable with four experimented phytochemicals such as (Z)-9,17octadecadienal, 2,3-dihydro-3,5-dihydroxy-6-methyl-4Hpyran-4-one, n-decanoic acid and n-hexadecanoic acid. These compounds were preferred by considering their retention time and medicinal assets. The phytocompounds had the potential to dock with the target proteins and their interaction details are listed in Table 2 and illustrated in Fig. 2. The target protein Monoamine oxidase (MAO) A was counteracted by three and (MAO) B with two reference compounds (Fig. 3), where kaempferol-3monoglucoside exhibited with a least score of $-13.90 /$ $-12.95 \mathrm{kcal} / \mathrm{mol}$. Binding studies was competitive among all the four test compounds the major compound (Z)-9,17octadecadienal obtained from the gas chromatogram assay with a high retention time (32.99) exhibited a minimum 
Fig. 2 a Interaction between kaempferol-3-monoglucoside and Mono Amine Oxidase A conducted by the amino acid residues ALA68, GLN443, GLY66, MET445, TYR69 and ASN181. b Interaction between Quercetin and Mono Amine Oxidase A conducted by the amino acid residues ASN181 and PHE 208. c Interaction between Malvidin-3-0glucoside and Mono Amine Oxidase A conducted by the amino acid residues ALA68, TYR69 and GLN215.

d Interaction between (Z)-9,17octadecadienal and monoamine oxidase A conducted by the amino acid residues ALA68 and TYR69. e Interaction between 2,3-dihydro-3,5dihydro-6-methyl-4H-pyran-4one and monoamine oxidase $\mathrm{A}$ conducted by the amino acid residue PHE 208. f Interaction between n-hexadecanoic acid and monoamine oxidase $\mathrm{A}$ conducted by the amino acid residues ALA68 and MET 445. g Interaction between n-decanoic acid and monoamine oxidase A conducted by the amino acid residues ALA68, MET 445 and TYR69
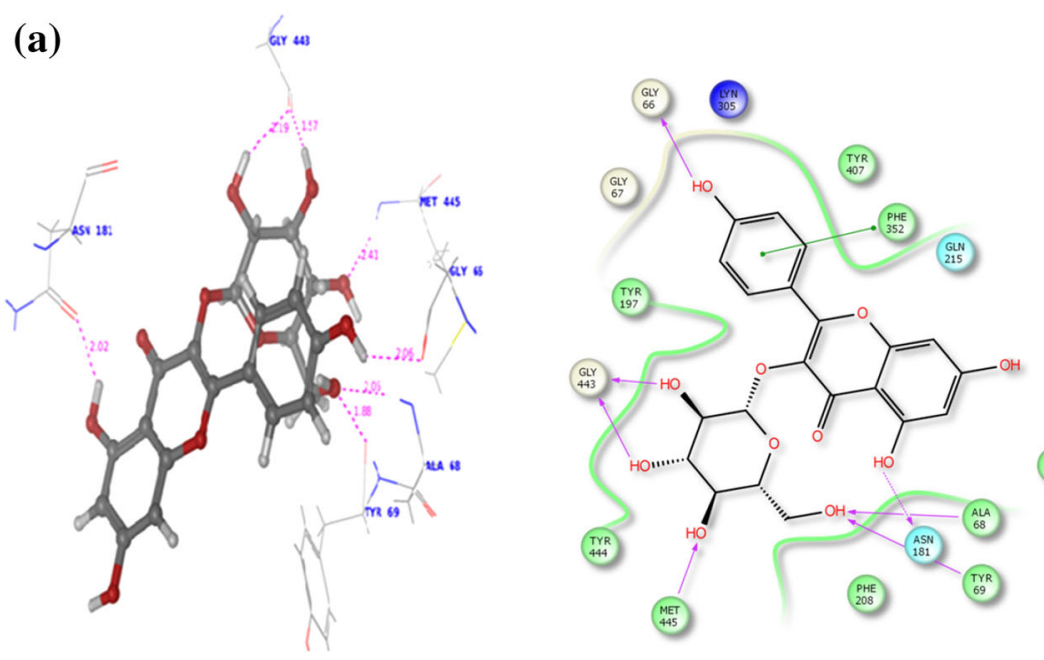

(b)
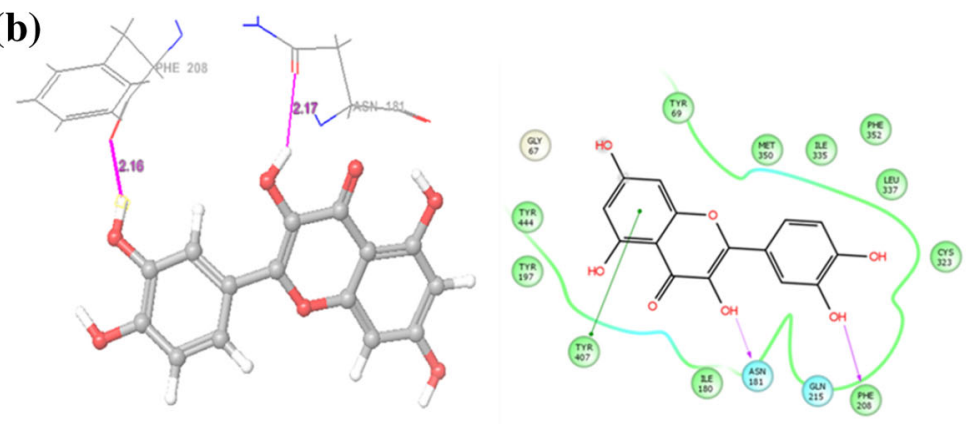

(c)
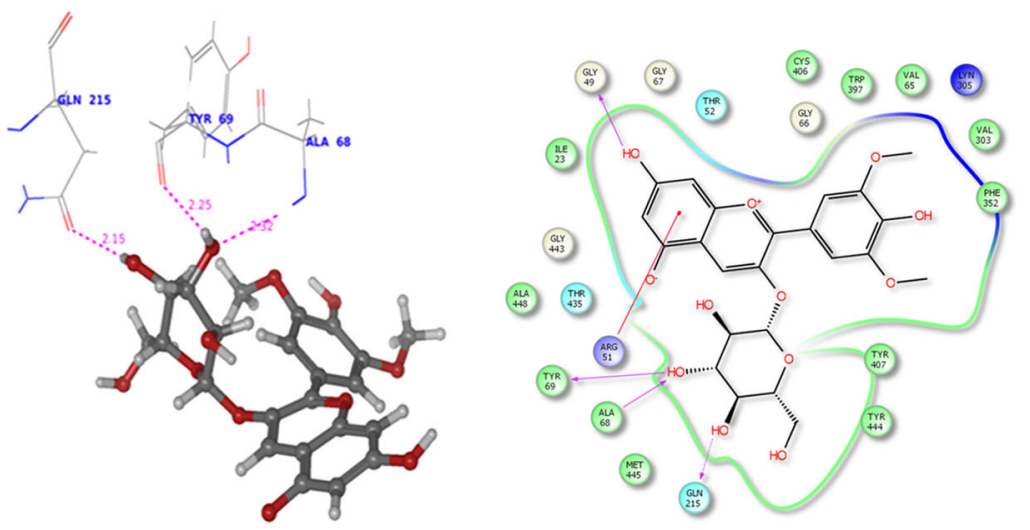

(d)

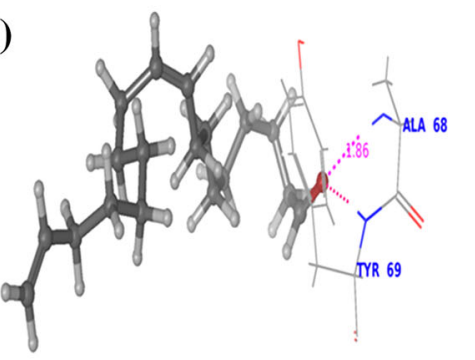

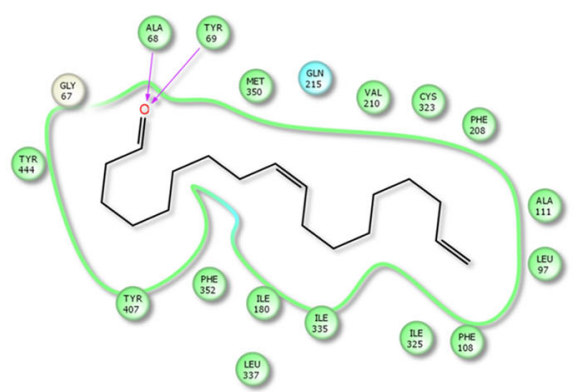


Fig. 2 continued (e)

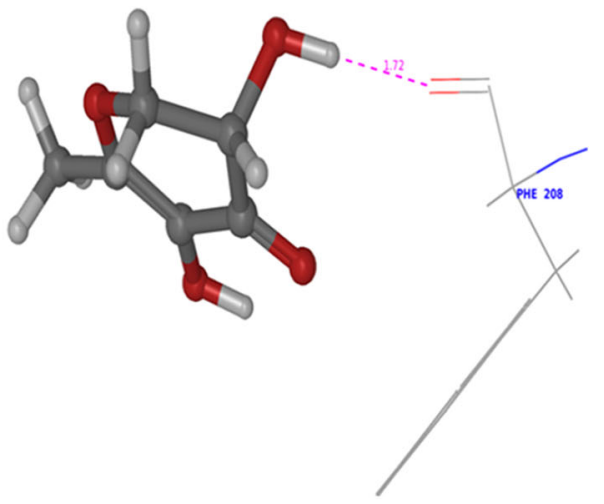

(f)

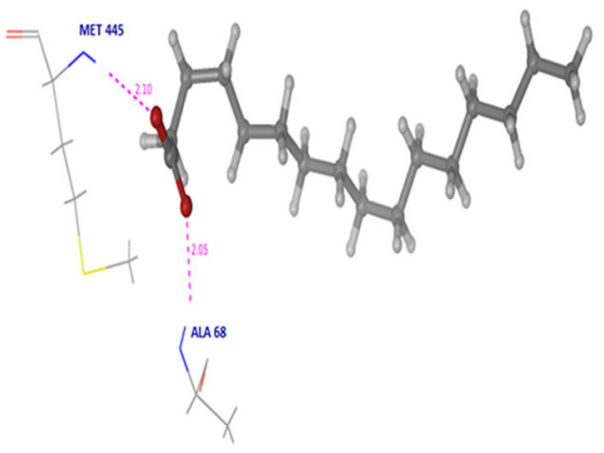

(g)

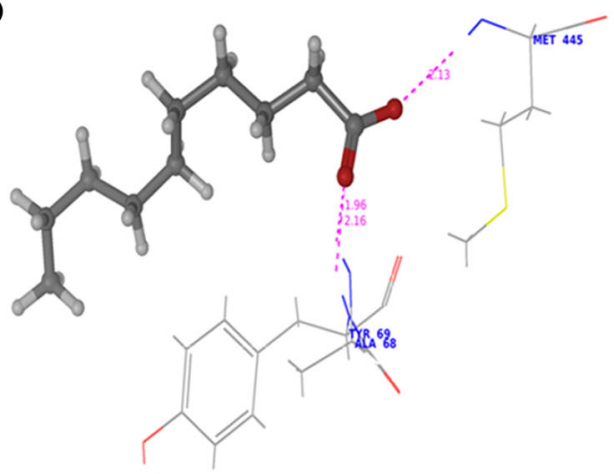

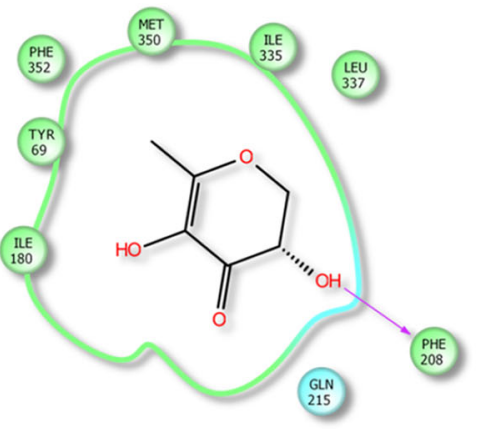
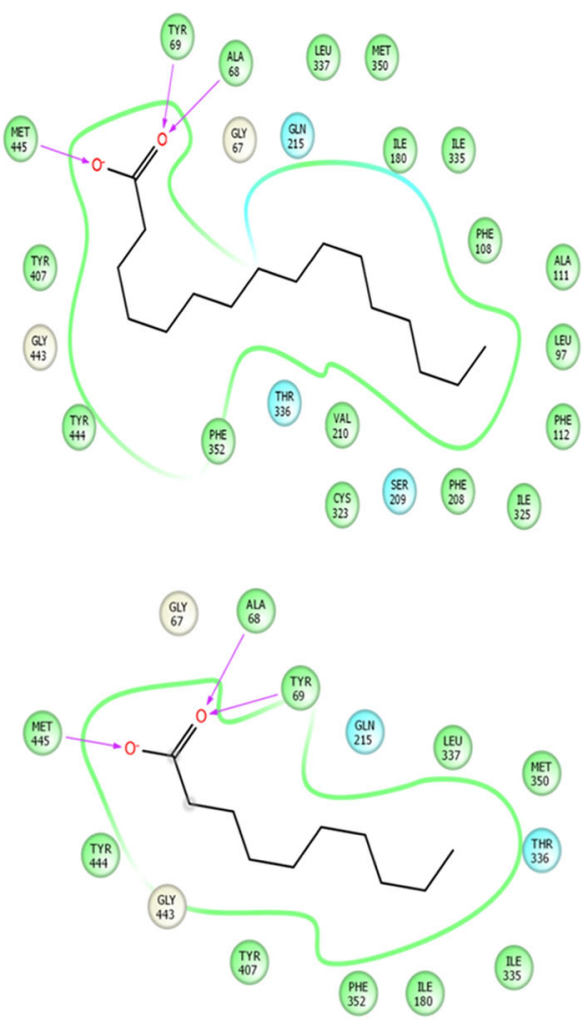

binding affinity energy values of $-6.5 /-7.71 \mathrm{kcal} / \mathrm{mol}$ against both the isoforms of monoamine oxidase (MAO) $\mathrm{A}$ and B. n-Hexadecanoic acid commonly known as palmitic acid conferred resistant against monoamine oxidase (MAO) B as depicted in Fig. 3c with a least docking score of $-10.5001 \mathrm{kcal} / \mathrm{mol}$. The active site associated with both the isoforms of monoamine oxidase is composed of amino acid residues, such as TYR 69, TYR 197 , PHE 208, GLU 216, TYR 407, PHE 352, TYR 444 (MAO) A and PHE 103, PRO 104, TRP 119, LEU 167, PHE 168, LEU 171, ILE 199, ILE 316 and TYR 326 (MAO) B [34, 39, 40]. The phytocompounds quercetin and 2,3-dihydro-3,5-dihydroxy-6-methyl-4H-pyran-4-one interacted with the active site of (MAO) A with the amino acid residue PHE 208. The study investigated diversity in the amino acid residues distinct from the active sites of both MAO A (ALA 68, GLN 443, GLN 66, MET 445, TYR 69 and ASN 181) and MAO B (LYS 296, TYR 60, GLY 434, MET 486, ILE 197, TYR 435 and CYS 172) (Table 3).

\section{Conclusion}

The present study provides an evidence for the extricated phytocompounds from the plant $C$. ternatea as new potent and selective hMAO-inhibitors. The results hoists two compounds, (Z)-9,17-octadecadienal and n-hexadecanoic 
Fig. 3 a Interaction between kaempferol-3-monoglucoside and monoamine oxidase $\mathrm{B}$ conducted by the amino acid residues LYS296, TYR60 and GLN434. b Interaction between quercetin and monoamine oxidase $\mathrm{B}$ conducted by the amino acid residue GLN434. c Interaction between n-hexadecanoic acid and monoamine oxidase $\mathrm{B}$ conducted by the amino acid residueTYR 60. d Interaction between $(Z)-9,17$ -

octadecadienal and monoamine oxidase B conducted by the amino acid residue MET 436. e Interaction between 2,3dihydro-3,5-dihydroxy-6methyl-4H-pyran-4-one and monoamine oxidase B conducted by the amino acid residue TYR 435 and ILE 198.

f Interaction between n-decanoic acid and monoamine oxidase $\mathrm{B}$ conducted by the amino acid residue CYS 172
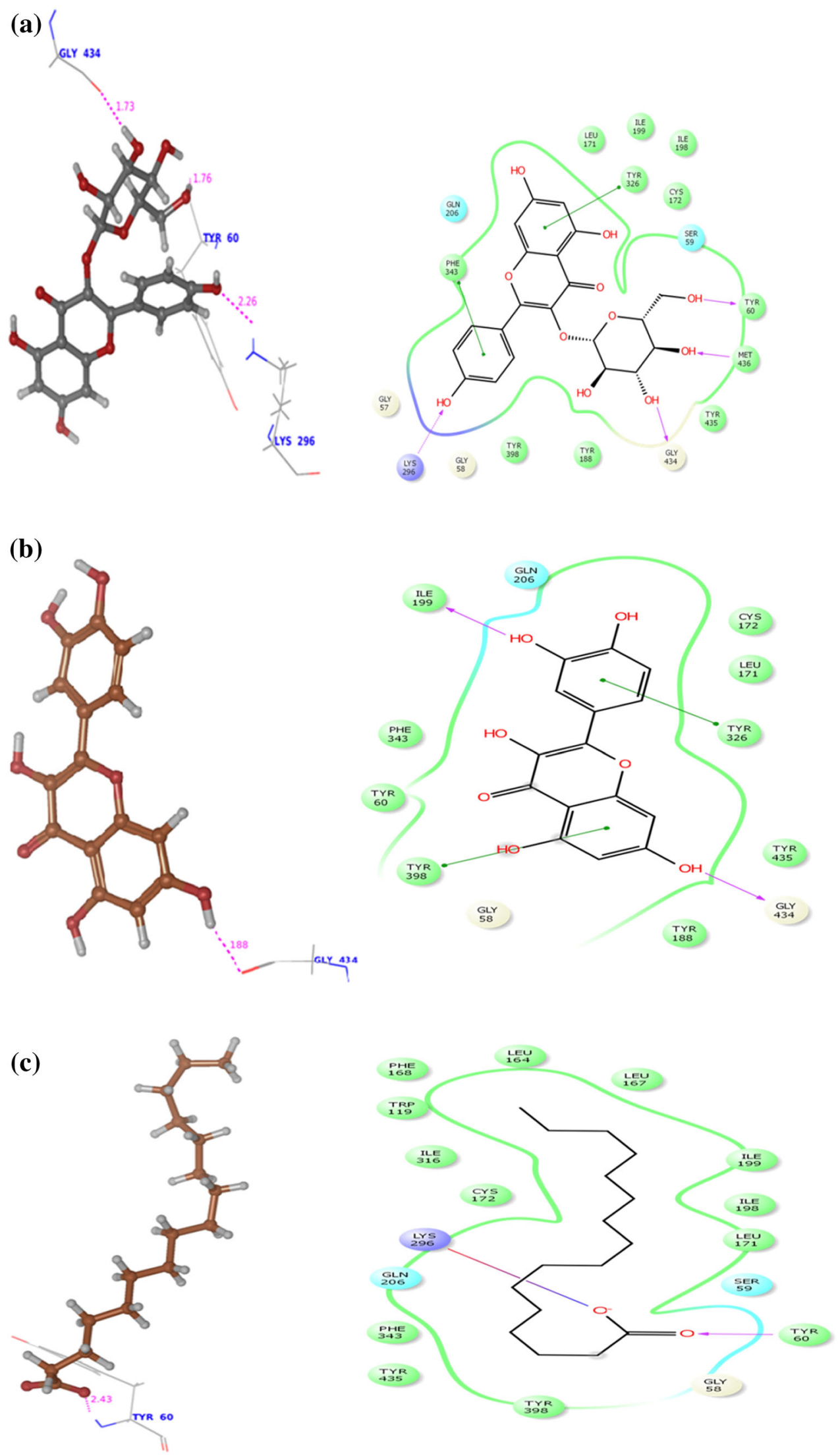
Fig. 3 continued

(d)

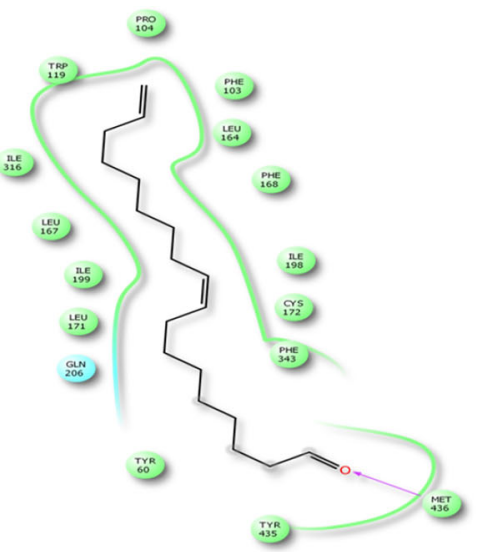

(e)

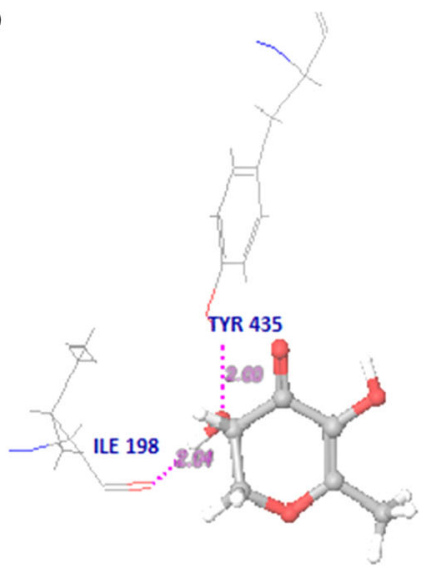

(f)

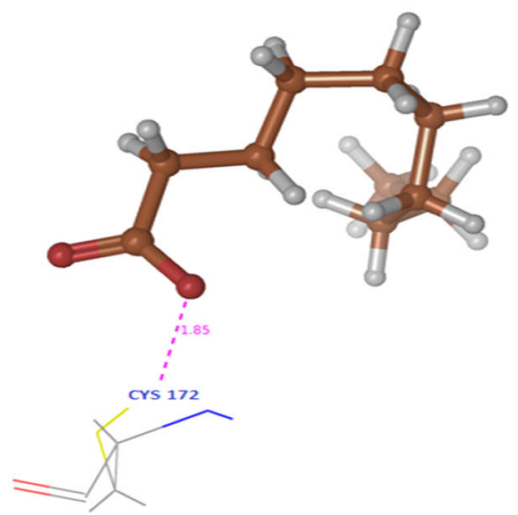

TYR
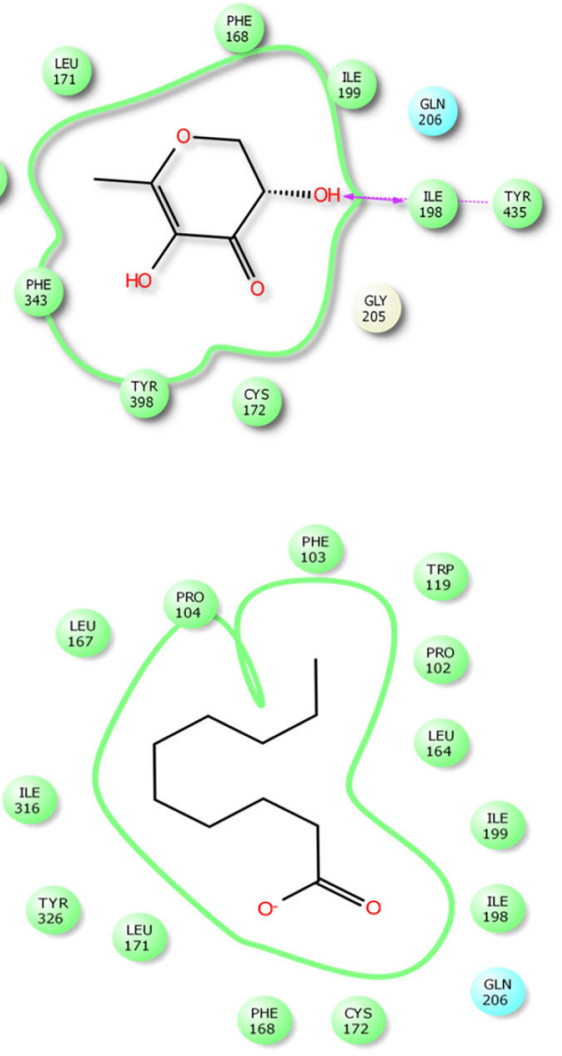

acid as potential lead molecules for developing novel selective MAO-A inhibitors which can confer herbal remedy in the treatment of psychiatric disorders such as depression, anxiety, and also cognitive impairments in Alzheimer's and Parkinson's Diseases. The in silico assay endorses the reference phyto compound Kaempferolmonoglucoside as an established brain drug. Further the study commences amino acid residues which are distinct from the residues pertaining to the active sites of monoamine Oxidase A and B. The efficacy of these potent phytocompounds can be further elucidated with clinical studies.

\section{Experimental Section}

\subsection{Chemicals}

All chemicals used were of analytical grade purchased from, sigma Aldrich Ltd.

\subsection{Plant Collection and Identification}

The healthy plant samples were collected from the premises of Bishop Heber College, Tiruchirappalli, India. The 
Table 3 Hit list of the ligands with the target protein MAO-B

\begin{tabular}{|c|c|c|c|c|c|}
\hline S. no & Compound & $\begin{array}{l}\text { Binding score } \\
\text { energy value } \\
(\mathrm{Kcal} / \mathrm{mol})\end{array}$ & $\begin{array}{l}\text { No. of hydrogen } \\
\text { bonds }\end{array}$ & $\begin{array}{l}\text { Hydrogen } \\
\text { bond length }\end{array}$ & $\begin{array}{l}\text { Interacting } \\
\text { amino acid } \\
\text { residue }\end{array}$ \\
\hline 1. & $\begin{array}{l}\text { Name: kaempferol-monoglucoside } \\
\text { Formula: } \mathrm{C}_{21} \mathrm{H}_{20} \mathrm{O}_{1} \\
\text { MW: } 448.3769 \\
\text { Pub Chem ID: } 5282102\end{array}$ & -12.9503 & 3 & $\begin{array}{l}2.25 \\
1.76 \\
1.73\end{array}$ & $\begin{array}{l}\text { LYS } 296 \\
\text { TYR } 60 \\
\text { GLY } 434\end{array}$ \\
\hline 2. & $\begin{array}{l}\text { Name: Quercetin } \\
\text { Formula: } \mathrm{C}_{20} \mathrm{H}_{40} \mathrm{O} \\
\text { MW: } 296.531 \\
\text { Pub Chem ID: } 5280343\end{array}$ & -10.637 & 1 & 1.88 & GLY 434 \\
\hline 3. & $\begin{array}{l}\text { Name: Malvidin-3-0-glucoside } \\
\text { Formula: } \mathrm{C}_{23} \mathrm{H}_{2}{ }_{5} \mathrm{O}_{12} \\
\text { MW: } 493.4374 \\
\text { Pub Chem ID: } 443652\end{array}$ & - & - & - & - \\
\hline 4. & $\begin{array}{l}\text { Name: Delphinidin-3,5-diglucoside } \\
\text { Formula: } \mathrm{C}_{27} \mathrm{H}_{31} \mathrm{O}_{17} \\
\text { MW: } 627.52484 \\
\text { Pub Chem ID: } 10100906\end{array}$ & - & - & - & - \\
\hline 5. & $\begin{array}{l}\text { Name: } n \text {-Hexadecanoic acid } \\
\text { Formula: } \mathrm{C}_{16} \mathrm{H}_{32} \mathrm{O}_{2} \\
\text { MW: } 256 \\
\text { Pub Chem ID: } 985\end{array}$ & -10.5001 & 1 & 2.43 & TYR 60 \\
\hline 6. & $\begin{array}{l}\text { Name: } 9,17 \text { Octadecadienal,(Z)- } \\
\text { Formula: } \mathrm{C}_{18} \mathrm{H}_{32} \mathrm{O} \\
\text { MW: } 264 \\
\text { Pub Chem ID: } 5365667\end{array}$ & -7.71444 & 1 & 2.01 & MET 436 \\
\hline \multirow[t]{2}{*}{7.} & $\begin{array}{l}\text { Name: 4H-Pyran-4-one, 2,3-dihydro- } \\
\text { 3,5-dihydroxy-6-methyl- } \\
\text { Formula: } \mathrm{C}_{6} \mathrm{H}_{8} \mathrm{O}_{4} \\
\text { MW: } 144 \\
\text { Pub Chem ID: } 119838\end{array}$ & -5.3433 & 2 & $\begin{array}{l}2.04 \\
2.00\end{array}$ & $\begin{array}{l}\text { ILE } 198 \\
\text { TYR } 435\end{array}$ \\
\hline & $\begin{array}{l}\text { 8. Name: n-Decanoic acid } \\
\text { Formula: } \mathrm{C}_{10} \mathrm{H}_{20} \mathrm{O}_{2} \\
\text { MW: } 172 \\
\text { Pub Chem ID: } 2969\end{array}$ & -4.29637 & 1 & 1.85 & CYS 172 \\
\hline
\end{tabular}

roots from the whole plant was segregated, cleaned and allowed to dry under the shade. The identification and voucher specimen number AAM 001 of the plant was sorted out and deposited at the Rapinat Herbarium and Centre for Molecular Systematic (St. Joseph's, College Tiruchirappalli, India). The authentication of the plant as C. ternatea L. was validated by Dr. S. John Britto (Director).

\subsection{Preparation of Plant Extract}

The shade dried powdered root sample (100 gm) was extracted with $250 \mathrm{~mL}$ of ethanol in a Soxhlet apparatus for $72 \mathrm{~h}$. The plant extract yielded was filtered and evaporated to dryness which was further used for analysis.

\subsection{Gas Chromatography-Mass Spectrometry Analysis}

GC-MS analysis of the plant extract was performed using a Perkin-Elmer Clarus 500 system comprising an AOC-20i auto-sampler. The Gas Chromatograph is interfaced to a Mass Spectrometer (GC-MS) equipped with a column (Id: $250 \mu \mathrm{m}$,) Elite-5MS (5\% diphenyl/95 \% dimethyl poly siloxane) fused extended to a length of $(30 \mathrm{~m})$. For GCMS detection, an electron ionization system was operated in electron impact mode with ionization energy of $70 \mathrm{eV}$. 
a

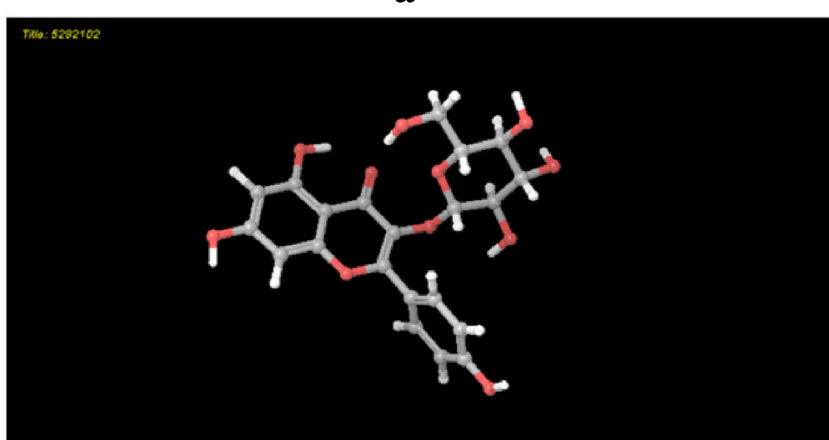

c

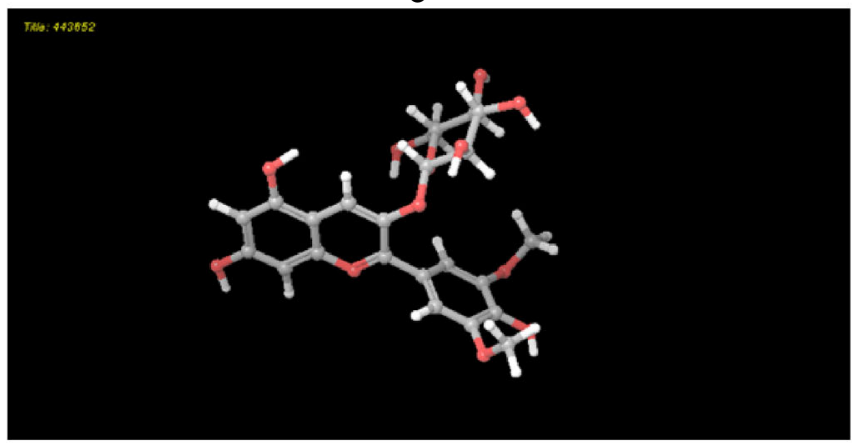

$\mathbf{e}$

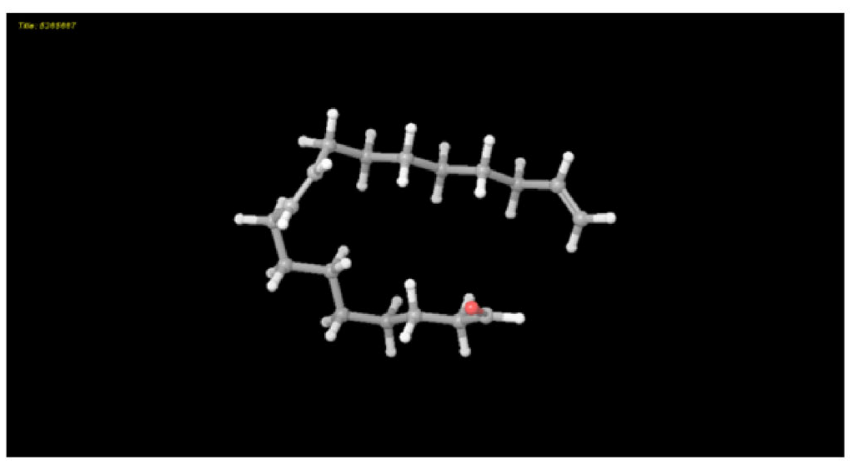

$\mathbf{g}$

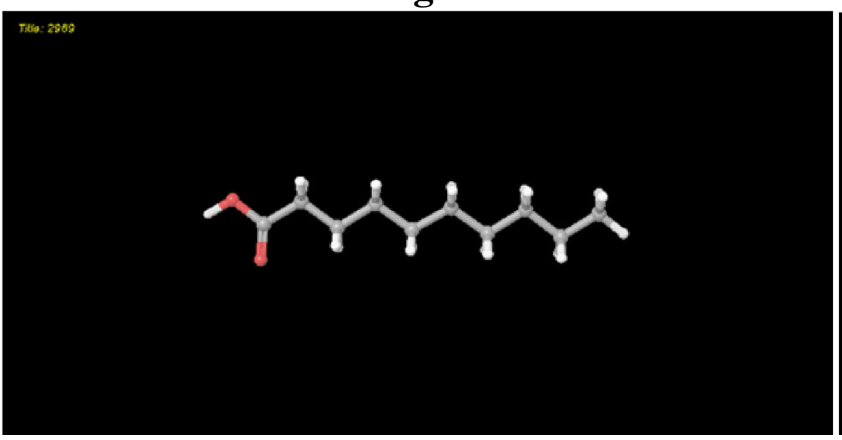

Fig. 43 D Structures of the phytochemicals as ligands. a kaempferol3-monoglucoside (CID: 5282102). b Delphinidin-3,5-diglucoside (CID:10100906). c Malvidin-3-0-glucoside(CID: 443652). d Quercetin b

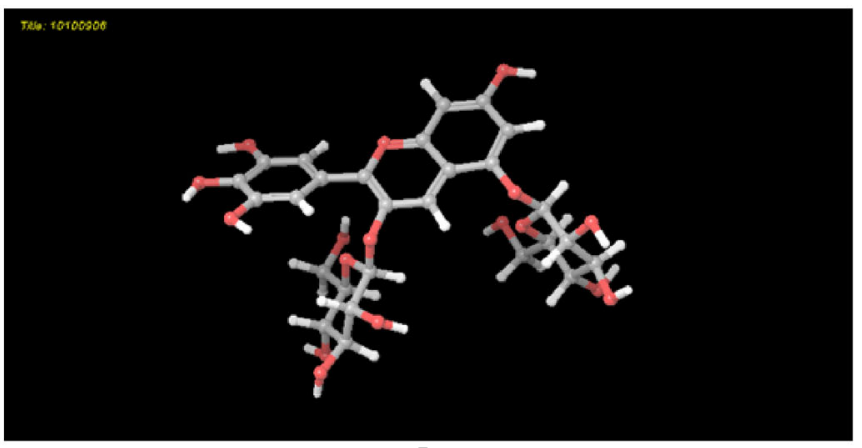

d

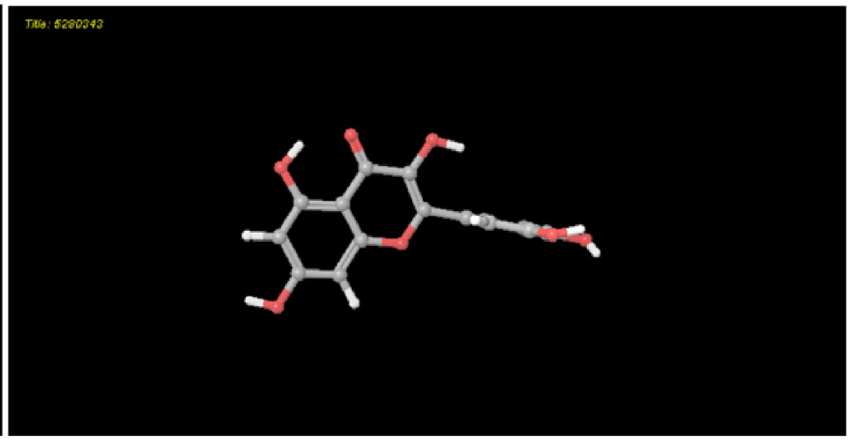

f

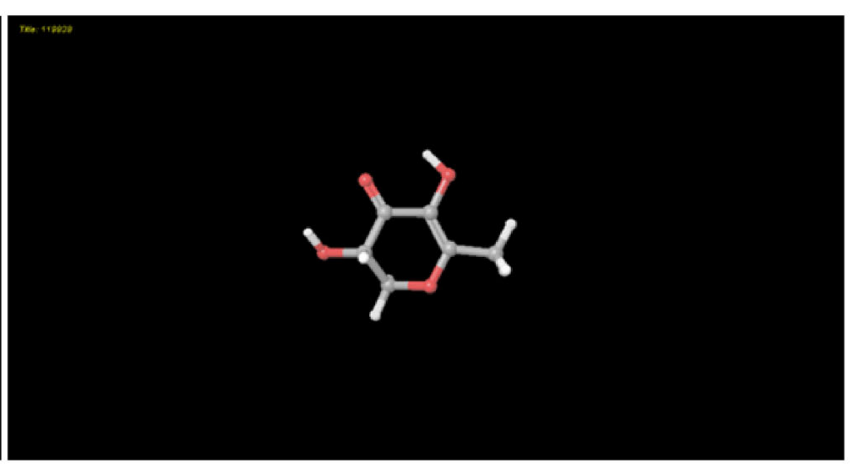

h

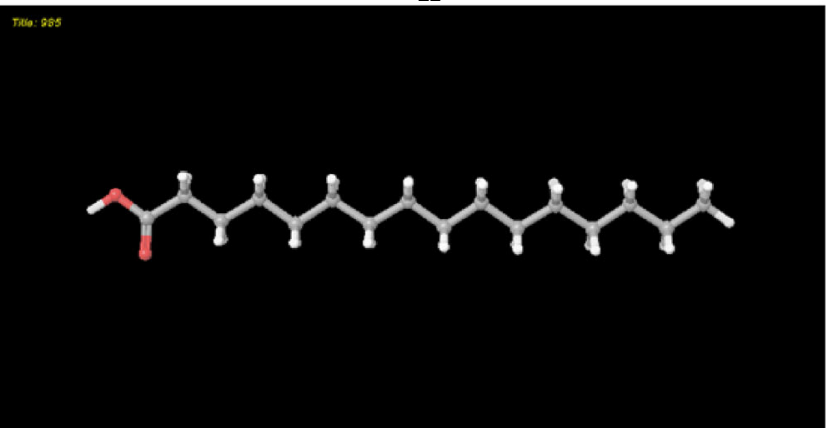

(CID: 5280343). e d.9,17-Octadecadienal, (Z)- (CID: 5365667). f. 4H-Pyran-4-one, 2,3-dihydro-3,5-dihydroxy-6-methyl (CID:119838). g n-Decanoic acid (CID: 2969). h n-Hexadecanoic acid (CID: 985) 
Helium gas $(99.999 \%)$ was used as a carrier gas at a constant flow rate of $1 \mathrm{ml} / \mathrm{min}$, and a sample injection volume of $1.6 \mu \mathrm{l}$ was employed (a split ratio of 10:1). The injector temperature was maintained at $280{ }^{\circ} \mathrm{C}$, the ion-source temperature was $200{ }^{\circ} \mathrm{C}$, the oven temperature was programmed from $60{ }^{\circ} \mathrm{C}$ (isothermal for $8 \mathrm{~min}$ ) which, increased to $200{ }^{\circ} \mathrm{C}$, for $5 \mathrm{~min}$ at $7{ }^{\circ} \mathrm{C}$ to $280{ }^{\circ} \mathrm{C}$, ending with $280{ }^{\circ} \mathrm{C}$ (isothermal) for $15 \mathrm{~min}$. Mass spectra were taken at $70 \mathrm{eV}$ at a scan interval of $0.5 \mathrm{~s}$ and fragments from 45 to $450 \mathrm{Da}$. The relative percentage amount of each component was calculated by comparing its average peak area to the total areas. The mass-detector used in this analysis was TurboMass Gold-Perkin-Elmer, with the software Turbo-Mass ver-5.2 to handle the mass spectra and chromatograms.

\subsection{Identification of Phytocomponents}

Interpretation on mass-spectrum GC-MS was conducted using the database of National Institute Standard and Technology (NIST) having more than 62000 patterns. The spectrum of the unknown components was compared with the spectrum of known components stored in the NIST library. The name, molecular weight, and structure of the components of the test materials were ascertained.

\subsection{Docking Analysis}

Molecular docking studies were carried to identify the binding affinities and interaction between the inhibitors and the target proteins (Mono Amine Oxidase) MAO-A and MAO-B using Glide software (Schrodinger Inc. U.S.A.Maestro version 10.2). Grid-based Ligand Docking with Energetic (Glide) is one of the most accurate docking tool available for ligand-protein, protein-protein binding studies. Glide was found to produce least number of inaccurate poses and $85 \%$ of Glides binding models had an RMSD of $1.4 \mathrm{~A}^{\circ}$ or less from native co-crystallized structures.

\subsection{Preparation of Ligands}

The 3-dimensional structures of the phytocompounds considered as ligands were retrieved and downloaded (Fig. 4a-h) as mol files from the site of Pub Chem. The molecules were processed using the LigPrep tool from Schrodinger to obtain the perfect conformation by the addition or removal of hydrogen atoms with respect to the OPLS_2005 force field.

\subsection{Preparation of Protein Target}

The target proteins Mono Amine Oxidase MAO-A and MAO-B were retrieved from Protein Data Bank (PDB). Water molecules were removed and a single chain was selected between two chains. Generally, all waters (except those coordinated to metals) are deleted, but water that connects between the ligand and the protein are sometimes retained. Problems in the PDB protein structure were repaired by adjusting the protein, metal ions, and cofactors. The structure forming bonds from the ligand or a cofactor to a protein metal were deleted by adjusting the ligand bond orders and formal charges. The minimization was done to restrain the input protein coordinates by a selected RMSD tolerance.

\subsection{GLIDE/Ligand Docking}

Grid generated output file was uploaded as an input for Ligand docking against protein prepared targets in GLIDE. SP (Standard Precision) mode was adopted. Flexible docking mode was selected.

Acknowledgments The authors are grateful to the Bishop Heber College and Jamal Mohamed College, Tiruchirappalli, Tamil Nadu, India for their support and encouragement. We thank Centre for Advanced Research in Indian System of Medicine (CARISM), of Sastra University, Tanjore, for their GC/MS studies and Department of Bioinformatics Bharathidasan University, Tiruchirappalli for guiding us in the in silico analysis.

\section{Compliance with Ethical Standards}

Conflict of interest The authors declare no conflict of interest.

Open Access This article is distributed under the terms of the Creative Commons Attribution 4.0 International License (http://creative commons.org/licenses/by/4.0/), which permits unrestricted use, distribution, and reproduction in any medium, provided you give appropriate credit to the original author(s) and the source, provide a link to the Creative Commons license, and indicate if changes were made.

\section{References}

1. P.K. Mukherjee, S. Rai, V. Kumar, K. Mukherjee, P.J. Hylands, R.C. Hider, Expert Opin. Drug Dis. 2, 633-657 (2007)

2. P.V. Sharma, Drauyaguna-Vijnana, vol. 2 (Chaukhambha Bharti Academy, Varanasi, 1998), pp. 3-29

3. K.C. Chuneker, Bhavaprakasa Nighantu, 6th edn. (Chaukhambha Bharti Academy, Varanasi, 1982), pp. 202-203

4. K.M. Nadkarni, Indian Materia Medica, vol. 1 (Popular Prakashan, Mumbai, 1976), pp. 9-10

5. A. Nahata, V.K. Dixit, Indian J. Pharm. Sci. 70, 834-837 (2008)

6. A. Nahata, U.K. Patil, V.K. Dixit, Nat. Prod. Res. 22, 1472-1482 (2008)

7. A. Nahata, U.K. Patil, V.K. Dixit, Pharm. Biol. 47, 444-451 (2009)

8. A. Nahata, U.K. Patil, V.K. Dixit, Phytother. Res. 24, 486-493 (2010)

9. N.K. Sethiya, A. Nahata, V.K. Dixit, Asian J. Tradit. Med. 3, 224-229 (2008)

10. V.V. Sivaranjan, I. Balachandran, Ayurvedic drugs and their plant sources (Oxford and IBH Publishing Company, New Delhi, 1994), pp. 425-428 
11. A.W.J. Bach, N.C. Lan, D.L. Johnson, C.W. Abell, M.E. Bembenek, S.W. Kwan, P.H. Seeburg, J.C. Shih, Proc. Natl. Acad. Sci. 85, 4934-4938 (1998)

12. F. Hubalek, C. Binda, A. Khalil, M. Li, A. Mattevi, N. Castagnoli, D.E. Edmondson, J. Biol. Chem. 280, 15761-15766 (2005)

13. M.B.H. Youdim, D.E. Edmondson, K.F. Tipton, Nat. Rev. Neurosci. 7, 295-309 (2006)

14. J.W. Buckholtz, A. Meyer-Lindenberg, Trends Neurosci. 31(3), 120-129 (2008)

15. H. Dorfman, A. Meyer-Lindenberg, J.W. Buckholtz, Curr. Top Behav. Neurosci. 75(1), 9-17 (2014)

16. K.A. Pavlov, D.A. Chistiakov, V.P. Chekhonin, J. Appl. Genet. 53(1), 61-82 (2012)

17. C.M. Pare, Pharmakopsychiatr. Neuropsychopharmakol. 9, 187-192 (1976)

18. J. Shih, K. Chen, M.J. Ridd, Annu. Rev. Neurosci. 22, 197-217 (1999)

19. C. Binda, F. Hubalek, M. Li, D.E. Edmondson, A. Mattevi, FEBS Lett. 564, 225-228 (2004)

20. J.W. Tetrud, J.M. Langston, Science 245, 519-522 (1989)

21. Anonymous, Indian Medicinal Plants, 2nd edn. (Orient Longman, Madras, 1995), pp. 129-132

22. A.D. Taranalli, T.C. Cheeramkuczhi, Pharm. Biol. 38, 51-56 (2000)

23. K.S. Rai, K.D. Murthy, K.S. Karanth, M.S. Rao, Indian J. Physiol. Pharmacol. 45, 305-315 (2001)

24. J.R. Hibbeln, The Lancet 351(9110), 1213 (1998)

25. M. Peet, B. Murphy, J. Shay, D. Horrobin, Biol. Psychiatry 43, 315-319 (1998)

26. B. Peet, C. Stokes, Drugs 65, 1051-1059 (2005)
27. G. Uma, V. Balasubramaniam, J. Chem. Pharm. Res. 4, 4417-4419 (2012)

28. D.A. Finn, A.S. Beadles-Bohling, E.H. Beckley, M.M. Ford, K.R. Gililland, R.E. Gorin-Meyer, K.M. Wiren, CNS Drug Rev. 12, 53-76 (2006)

29. F.V. DeFeudis, K. Drieu, Drug Dev. Res. 62, 1-25 (2004)

30. P.C. Chan, Q.S. Xia, P.P. Fu, J. Environ. Sci. Health C Environ. Carcinog. Ecotoxicol. Rev. 25, 211-244 (2007)

31. C.J. Fehske, K. Leuner, W.E. Muller, Pharmacol. Res. 60, 68-73 (2009)

32. L. Ren, F. Wang, Z. Xu, W.M. Chan, C. Zhao, H. Xue, Biochem. Pharmacol. 79, 1337-1344 (2010)

33. K. Dhawan, S. Dhawan, A. Sharma Passiflora, J. Ethnopharmacol. 94, 1-23 (2004)

34. D.H. Kim, S.J. Jeon, K.H. Son, J.W. Jung, S. Lee, B.H. Yoon, J.J. Lee, Y.W. Cho, J.H. Cheong, Neurobiol. Learn. Mem. 87, 536-546 (2007)

35. V. Vingtdeux, U. Dreses-Werringloer, H. Zhao, P. Davies, P. Marambaud, BMC Neurosci. 9, S2-S6 (2008)

36. D. Commenges, V. Scotet, S. Renaud, H. Jacqmin-Gadda, P. Barberger-Gateau, J.F. Dartigues, Eur. J. Epidemiol. 16, 357-363 (2000)

37. F.P. Turkmenoglu, İ. Baysal, S.C. Yabanoglu, K. Yelekci, H. Temel, S. Paşa, N. Ezer, İ. Çalış, G. Ucar, Molecules 20, 7454-7473 (2015)

38. K. Kazuma, K. Kogawa, N. Noda, N. Kato, M. Suzuki, Chem Biodivers. 1, 1762-1770 (2004)

39. K. Kazuma, N. Noda, M. Suzuki, Phytochemistry 62, 229-237 (2003)

40. T. Kondo, M. Ueda, T. Goto, Tetrahedron Lett. 46, 4749-4756 (1990) 